and children: guidelines by the Surgical Infection Society and the Infectious Diseases Society of America. Clin Infect Dis 2010;50:133-164.

9. Lopez-Cerero L, Picon E, Morillo C, et al. Comparative assessment of inoculum effects on the antimicrobial activity of amoxycillinclavulanate and piperacillin-tazobactam with extended-spectrum $\beta$-lactamase-producing and extended-spectrum $\beta$-lactamase-nonproducing Escherichia coli isolates. Clin Microbiol Infect 2010;16:132-136.

10. Tamma PD, Girdwood SC, Gopaul R, et al. The use of cefepime for treating AmpC $\beta$-lactamase-producing Enterobacteriaceae. Clin Infect Dis 2013;57:781-788.

\section{Transmission of ST8-USA300 Latin American Variant Methicillin-Resistant Staphylococcus aureus on a Neonatal Intensive Care Unit: Recurrent Skin and Soft- Tissue Infections as a Marker for Epidemic Community-Associated- MRSA Colonization}

To the Editor-We report a mother-to-newborn transmission of ST8-USA300 Latin American Variant methicillin-resistant Staphylococcus aureus (MRSA) on a neonatal intensive care unit during kangaroo mother care in a German University Hospital, which raises the question of whether recurrent skin and soft-tissue infection (SSTI) is an important marker for colonization with epidemic MRSA clones. The clonal expansion of the particularly virulent MRSA strain pulsotype USA300 is much dreaded. USA300 is the predominant MRSA clone circulating in the community in the United States, and it is recognized as a common cause of nosocomial $S$. aureus bloodstream infections, increasingly blurring the classic distinction between community- and hospital-associated MRSA. ${ }^{1}$ A variant of this virulent strain, designated as the Latin American Variant of USA300 MRSA (USA300-LV), was able to infiltrate, disseminate, and become the predominant MRSA in the community as well as in healthcare settings across most of Latin America. ${ }^{2}$

Although international travel and migration fosters the global spread of $S$. aureus, ${ }^{3,4}$ detection of USA300-LV in Europe is rare $\mathrm{r}^{1,3}$ and generally occurs in subjects with close family or travel links to Latin America. ${ }^{3}$ Nevertheless, the first observations of its autochthonous spread in the community have been reported in Spain and Italy. ${ }^{1}$ To date, in-hospital transmission of USA300-LV is an entirely unknown phenomenon in Europe, in contrast to Latin America, where it accounts for a substantial proportion of the nosocomial MRSA infections in Columbia and Uruguay. ${ }^{1,2}$

In October 2016, preoperative screening revealed PantonValentine leucocidin-positive (PVL+) MRSA isolated from a nasal swab of a 16-day-old, premature newborn (gestational age 33 weeks 2 days; $1,360 \mathrm{~g}$ ) that had been hospitalized since birth due to a congenital heart anomaly (index case) (Table 1). Two days later, MRSA was also detected in the nose and breast milk of the mother, who at that time provided kangaroo mother care (ie, skin-to-skin care) to her child on a daily basis. ${ }^{5}$ Because a postpartum screening of the child had been negative for multidrug-resistant organisms and because no other patients with MRSA colonization or infection had been treated on the same unit at that time, transmission from the mother is the most likely source of USA300-LV in the newborn. This finding is in line with research from Japan that found kangaroo mother care on the neonatal intensive care unit (NICU), although perceived to protect against infectious disease outcomes by increasing the diversity of the baby's microbiome, ${ }^{5}$ to be associated with 3.82 -fold increased odds of MRSA infection (95\% confidence interval, $1.11-13.13){ }^{6}$

Searching for the source of MRSA, a medical history among family members revealed recurrent SSTIs in the father and the 4-year-old sister of the index case patient. Swabs of the father's nose and from a resolving purulent SSTI on the sister's leg screened positive for PVL + MRSA. Although decolonization measures were immediately initiated, the mother's cesarean section wound became infected and PVL + MRSA USA300-LV could be cultured from the wound and stitch on day 24 after birth.

All family members received immediate MRSA eradication treatment according to the institutional protocol. The child was isolated for the rest of the hospital stay. Active screening did not detect transmissions to other patients on the same ward. No further cases of MRSA infection have occurred on the NICU unit since October 2016.

All strains isolated were of the spa type t008 (ST8), PVL positive, arginine catabolic mobile element (ACME) negative and bear the SCCmec type IVc, which is consistent with the ST8-USA300-LV MRSA clone. None of the family members reported significant travel outside Europe in the last 24 months, in particular, not to the United States or Latin America. However, the father of the index case had returned from a trip to Spain more than 12 months previously and prior to suffering from recurrent SSTI.

Among other risk factors, current guidelines recommend screening of intercontinental travelers and patients with active skin infections, prior hospitalization, or contact to patients carrying multidrug-resistant organisms (MDRO) for carriage of MDRO upon admission. ${ }^{7,8}$ In the presented case, the mother of the index patient did not fulfill any of the locally implemented criteria and was thus not screened on admission for C-section. Hence, to increase the sensitivity of CA-MRSA detection in the future, we propose targeted screening of all patients reporting recurrent SSTI defined as 2 or more episodes within the last 12 months in either (1) themselves or (2) members of the same household. This rationale is supported by reports on (1) recurrent skin infection being linked 


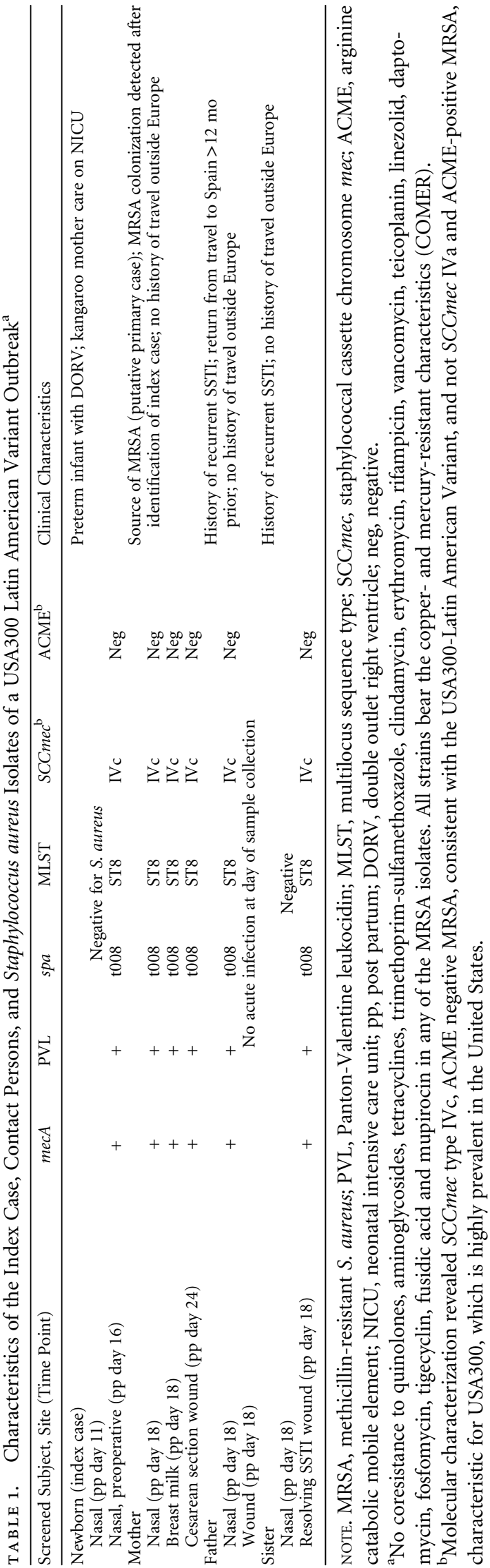

to PVL-positive S. aureus nasal colonization ${ }^{3,4}$ and (2) nosocomial outbreaks of epidemic PVL + CA-MRSA other than USA300 being fueled by colonized individuals that suffer from recurrent SSTI. ${ }^{9}$ Although the impact of the proposed approach has not been systematically evaluated, these observations in conjunction with our report suggests that a history of recurrent SSTI is a potent marker for colonization with epidemic CAMRSA and could thus prove useful for its early detection and timely eradication in newly admitted patients before invasion of epidemic CA-MRSA into European hospitals becomes an unchangeable fact. A recent report of a USA300 outbreak in a Belgium hospital demonstrates the urgency of this issue ${ }^{10}$ and justifies taking measures before direct evidence is available. Finally, European surveillance activities must address the silent invasion by USA300-LV and must monitor the import and spread of ACME-neg, MLST 8, SCCmec IVc MRSA.

\section{ACKNOWLEDGMENTS}

Financial support: No financial support was provided relevant to this article.

Potential conflicts of interest: All authors report no conflicts of interest relevant to this article.

\section{Dennis Nurjadi, MD; ${ }^{1}$ Sabrina Klein, MD; ${ }^{1}$ Stefan Zimmermann, MD; ${ }^{1}$ Klaus Heeg, MD; ${ }^{1}$ Philipp Zanger, $\mathrm{MD}^{1,2}$}

\begin{abstract}
Affiliation: 1. Department of Infectious Diseases, Heidelberg University Hospital, Heidelberg, Germany; 2. Institute of Public Health, Heidelberg University Hospital, Heidelberg, Germany.

Address correspondence to Dennis Nurjadi, MD, Heidelberg University Hospital, Department of Infectious Diseases, Im Neuenheimer Feld 324, Heidelberg 69120, Germany (dennis.nurjadi@uni-heidelberg.de). Infect Control Hosp Epidemiol 2017;38:883-885

(C) 2017 by The Society for Healthcare Epidemiology of America. All rights reserved. 0899-823X/2017/3807-0023. DOI: 10.1017/ice.2017.88
\end{abstract}

\section{REFERENCES}

1. Nimmo GR. USA300 abroad: global spread of a virulent strain of community-associated methicillin-resistant Staphylococcus aureus. Clin Microbiol Infect 2012;18:725-734.

2. Reyes J, Rincon S, Diaz L, et al. Dissemination of methicillinresistant Staphylococcus aureus USA300 sequence type 8 lineage in Latin America. Clin Infect Dis 2009;49:1861-1867.

3. Nurjadi D, Friedrich-Janicke B, Schafer J, et al. Skin and soft tissue infections in intercontinental travellers and the import of multi-resistant Staphylococcus aureus to Europe. Clin Microbiol Infect 2015;21:567e1-e10.

4. Zanger P, Nurjadi D, Schleucher R, et al. Import and spread of Panton-Valentine Leukocidin-positive Staphylococcus aureus through nasal carriage and skin infections in travelers returning from the tropics and subtropics. Clin Infect Dis 2012;54:483-492.

5. Conde-Agudelo A, Diaz-Rossello JL. Kangaroo mother care to reduce morbidity and mortality in low birthweight infants. Cochrane Database Syst Rev 2016. doi: 10.1002/14651858.CD002771. 
6. Sakaki H, Nishioka M, Kanda K, Takahashi Y. An investigation of the risk factors for infection with methicillin-resistant Staphylococcus aureus among patients in a neonatal intensive care unit. Am J Infect Control 2009;37:580-586.

7. Recommendations for prevention and control of methicillinresistant Staphylococcus aureus (MRSA) in medical and nursing facilities [in German]. Bundesgesundheitsblatt Gesundheitsforschung Gesundheitsschutz 2014;57:696-732.

8. Calfee DP, Salgado CD, Classen D, et al. Strategies to prevent transmission of methicillin-resistant Staphylococcus aureus in acute care hospitals. Infect Control Hosp Epidemiol 2008;29(Suppl 1):S62-S80.

9. Orendi JM, Coetzee N, Ellington MJ, et al. Community and nosocomial transmission of Panton-Valentine leucocidin-positive community-associated meticillin-resistant Staphylococcus aureus: implications for healthcare. J Hosp Infect 2010;75:258-264.

10. Kairet K, Ho E, Van Kerkhoven D, et al. USA300, A strain of community-associated methicillin-resistant Staphylococcus aureus, crossing Belgium's borders: outbreak of skin and soft tissue infections in a hospital in Belgium. Eur J Clin Microbiol Infect Dis 2017. doi: 10.1007/s10096-016-2883-6.

\section{Multiple Norovirus Outbreaks Due to Shredded, Dried, Laver Seaweed in Japan}

To the Editor-Seaweed has been an important traditional Japanese food, and various types of seaweed are widely used in soup stock, seasonings, and sushi dishes. The history of seaweed consumption is long and deeply rooted in Japanese tradition; remains of marine algae were even found in some prehistoric relics in the Japanese archipelago. Interestingly, comparative metagenome analyses of gut microbiota have suggested the presence of a unique microbiome derived from marine bacteria related to seaweed consumption in Japanese individuals. ${ }^{1}$ Among seaweeds, shredded, dried, laver seaweed is commonly used as a condiment; previously, it has rarely been considered to cause food poisoning. Here, we report unprecedented multiple norovirus outbreaks due to shredded, dried, laver seaweed packaged for long-term preservation in Japan.

Beginning on February 17, 2017, dozens of patients, mainly primary-school students, were treated in our clinic in Tachikawa City in Tokyo (Figure 1). A norovirus outbreak was responsible for illness in this unusually large cohort of patients. ${ }^{2,3}$ Ultimately, this outbreak included $\sim 1,100$ students and teachers from 7 primary schools in Tachikawa. All schools receive school lunches from the same centralized school lunch kitchen.

Although neither norovirus nor other pathogens were identified among cooking staff or in the kitchen facilities, the Tokyo metropolitan office identified the strain of norovirus that affected the outbreak patients in several packs of shredded, dried, laver seaweed used as condiments for the school lunches. ${ }^{2,3}$ The seaweed had been harvested in western Japan, Hyogo Prefecture. After being processed in a facility in Osaka

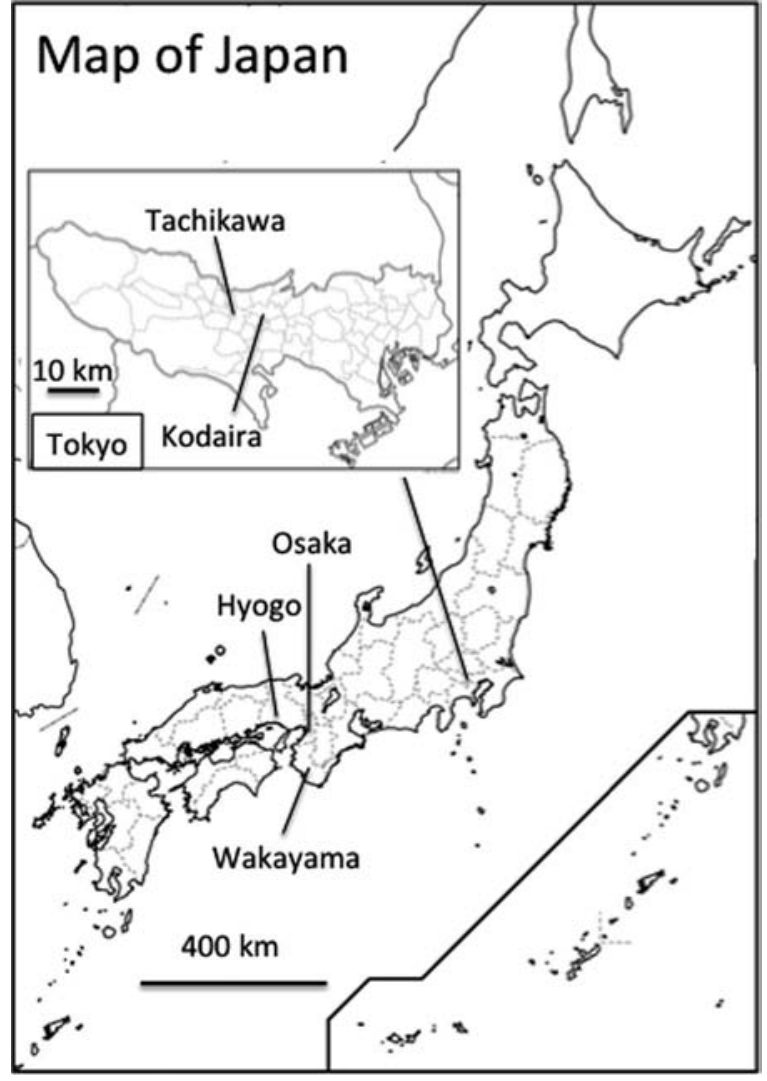

FIGURE 1. Geographical locations related to the multiple norovirus outbreaks due to shredded, dried, laver seaweed in Japan.

Prefecture, it was shipped nationwide with a December 2017 expiry date.

The company stated that the seaweed was heat treated using a conveyor-type machine for $\sim 7$ seconds at $240^{\circ} \mathrm{C}$ after boiling for 2 hours at $90{ }^{\circ} \mathrm{C}$. An infected subcontractor had handled the seaweed with bare hands during the subsequent cutting and packaging process in the factory. In addition, 2 similar outbreaks had occurred in January 2017 involving $>800$ and $>100$ school children, respectively, in geographically distant cities, Wakayama Prefecture and Kodaira City in Tokyo. In both outbreaks, seaweed from the same company contaminated with the same norovirus strain was provided in the school lunches. ${ }^{4}$

Noroviruses are surprisingly resistant to harsh environmental conditions, and they are widely recognized as the leading cause of viral gastroenteritis due to foodborne infection across all age groups. ${ }^{5-7}$ The diagnosis of norovirus gastroenteritis cannot be made based on clinical features alone, and the widespread availability of molecular diagnostic tests has resulted in an increased awareness of norovirus-associated acute gastroenteritis worldwide. Because no vaccines or specific antiviral agents are currently available for the prevention or treatment of norovirus infection, rigorous hygiene (eg, hand washing) and heat treatment of foods and water remain the main prevention measures to combat norovirus 